\title{
Using Data Provenance to Measure Information Assurance Attributes
}

\author{
Abha Moitra ${ }^{1}$, Bruce Barnett ${ }^{1}$, Andrew Crapo ${ }^{1}$, and Stephen J. Dill ${ }^{2}$ \\ ${ }^{1}$ General Electric Global Research, 1 Research Circle, Niskayuna, NY 12309 \\ \{moitraa, BarnettBr, Andrew. Crapo\} @ge.com \\ ${ }^{2}$ Lockheed Martin IS\&GS, \\ 321 Ballenger Center Drive, MS 125/1F10, Frederick, MD \\ Stephen.J.Dillalmco.com
}

\begin{abstract}
Data Provenance is multi-dimensional metadata that specifies Information Assurance attributes like Confidentiality, Authenticity, Integrity, Non-Repudiation etc. It may also include ownership, processing details and other attributes. Further, each Information Assurance attribute may itself have sub-components like objective and subjective values or application security versus transport security. Traditionally, the Information Assurance attributes have been specified probabilistically as a belief value (or corresponding disbelief value) in that Information Assurance attribute. In this paper we introduce a framework based on Subjective Logic that incorporates uncertainty by representing values as a triple of <belief, disbelief, uncertainty>. This framework also allows us to work with conflicting Information Assurance attribute values that may arise from multiple views of an object. We also introduce a formal semantic model for specifying and reasoning over Information assurance properties in a workflow. Data Provenance information can grow substantially as the amount of information kept for each object increases as well as the complexity of a workflow increases. In such situations, it may be necessary to summarize the Data Provenance information. Further, the summarization may depend on the Information Assurance attributes as well as the type of analysis used for Data Provenance. We show how such summarization can be done and how it can be used to generate trust value in the data. We also discuss how the Information Assurance values can be visualized.
\end{abstract}

Keywords: data provenance, information assurance, subjective logic, semantic model, uncertainty framework, Figure of Merit, trust, confidence.

\section{Introduction}

Our primary interest is in calculating the assurance in data. Components used to calculate this are the Information Assurance (IA) communication attributes, which include attributes of confidentiality, integrity, authenticity, non-repudiation, and availability. Factors that impact this include opinions of the data sources and of the certificate authorities used during the authentication process. These values are based on the observer's viewpoint, loyalties, and knowledge, and are therefore highly subjective. 
For simplicity we will not address these factors in this paper. Instead, we will focus on the information assurance attributes of the communication itself, related to the communication channel and process. If all parties agree on the relative strength of cryptographic algorithms at a certain point in time, then this forms the basis for an objective and consistent measurement of information assurance values across multiple parties regarding a set of messages.

In this paper we describe a model of information flow based on simple and complex messages (messages with attachments) about which objective information assurance attribute values are collected. This model includes the capability to rollup data provenance information over a complex message and/or over a multi-step information flow. We call these aggregations a Figures of Merit or FoM.

Given objective information assurance attribute values for a message or a figure of merit, our next goal is to summarize these in a simple visual icon that allows those who must act on information quickly to understand how confidential, authentic, and unmodified the data is, therefore allowing them to make more educated choices when dealing with the data.

\section{Previous Work}

In our previous work [1], we developed a generalized and flexible framework that was independent of any implementation, yet allowed a series of data provenance records to be captured, and analyzed. We summarize the framework below.

Each time a message is moved between agents, systems or processes, a single Data Provenance (DP) record is created. This record might be stored or sent along in parallel with the message. During the analysis, all of the records related to a single message are assumed to be available. Each DP record has two parts: one from the sender and one from the receiver.

Each part has an invariant and a variant section. The variant section may contain routing information to forward the message to the final destination, and may change during the routine process. The invariant part remains unchanged, allowing cryptographic hashes of this section to be consistent. The sender's invariant section may include the following components:

- Identity of the Author of the message

- Message ID

- Timestamp

- Message contents and type

- References to other message IDs, e.g., attachments

- Destination

- Security label or classification

- Outgoing Information Assurance values

It also includes the hash value (and name of hash algorithm) of the message contents. The sender (or someone acting as a proxy) may optionally sign the DP record, or attach a hash encrypted with a private key of the record, as assurance the DP record has not been modified. 
The receiver appends his own values to the record, adding

- Identity of the Receiver of the message

- Timestamp

- Incoming Information Assurance values

- Hash of the message body as seen by the receiver

The receiver may append a signature or an encrypted hash based on both the sender and receiver's records. One important characteristic of this approach is that third parties can validate the DP record at a later date.

There are several possible variations of the format. The timestamp may be part of the message ID. Some systems may split the message body from the record, and retrieve it by message ID when validating the record. Also, the message may be encrypted and the receiver may not have the key, as it may be forwarding the encrypted message to a third party. The receiver can create a record that they received an encrypted message, and provide a signed hash as proof. DP records can also have multiple signatures from multiple parties, if desired. This is useful in multiple trust domains. It is also possible that some of these fields may be blank, as the sender may not have any encryption capabilities.

Using this foundation, we build a system that implements this framework. We then developed a mechanism to calculate the information assurance attributes based on the DP records that are available.

\section{Subjective Logic}

We needed a flexible mechanism to calculate confidence that also allowed us to deal with uncertainty. We used Jøsang's Subjective Logic [2], which uses three values $b$, $d$, and $u$, where:

$b=$ belief, or the belief that the proposition is true

$d=$ disbelief, or the belief the proposition is false

$u=$ uncertainty, or the amount of uncommitted belief

These components satisfy $b+d+u=1$, and $b, d, u \in[0,1]$

\section{Implementation Details}

In order to make our prototype implementation more transparent to both our team members and to our sponsor, we chose to capture our models of information flow and of the data provenance at each point along the flow in a semantic model rather than in a traditional programming language. Our target representation was the Web Ontology Language (OWL) with a rules layer to capture domain inferences not implied by the formal models. We used a controlled English representation called the Semantic Application Design Language (SADL) as the authoring environment [6]. SADL is a language that maps directly and unambiguously into OWL and Jena Rules or SWRL. An Eclipse-based SADL-IDE supports the authoring, testing, and version control of the models. 
Information flow in our prototype is represented as instances of Message passed between instances of Agent. Snapshots of the data provenance state of the Message are captured as instances of DPInfo. When an Agent sends a Message, a SenderDPInfo (subclass of DPInfo) captures relevant data provenance information. When an Agent receives a Message, a ReceiverDPInfo (also a sub class of DPInfo) captures the data provenance state at receipt. There is an association between these two DPInfo instances and the process of moving a Message from a sending Agent to a receiving Agent is called a Hop. A series of Hops is called a Flow.

The definitions of DPInfo, SenderDPInfo, and ReceiverDPInfo are shown in Figure 1 in SADL syntax. Note that common attributes include measures of Integrity, Confidentiality, and Authenticity expressed as Subjective Logic values.

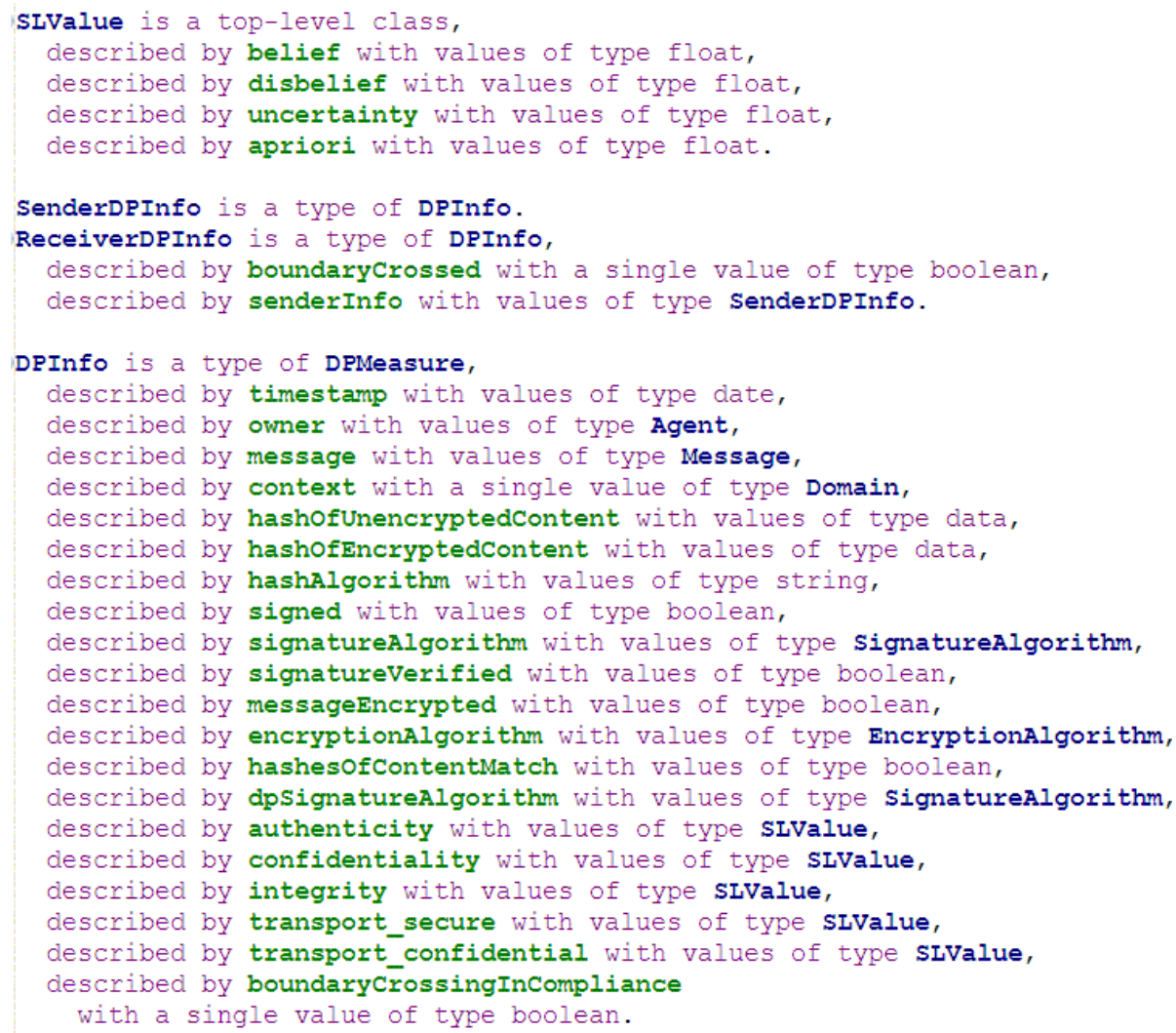

Fig. 1. Definition of DPInfo in SADL

Messages can be complex, meaning that a Message can include Attachments (subclass of Message), which can in turn have Attachments, etc. For a given Hop, the toplevel Message, which is not an Attachment at that point in the Flow, is referred to as the root container. It is desirable to roll up information across the parts of a complex message to obtain a single composite representation of the data provenance state of 
the whole Message. We call such a composite view a Figure Of Merit. Note that while a Figure Of Merit is a simplifying aggregation, which can more easily be perceived by a human observer, the DPInfo instances used in the rollup will normally be available so that drilldown into details is possible if desired. Note that we were measuring attributes of the transmission of the information, and did not measure attributes related to the contents of the messages. We considered the contents to be opaque. We also did not address contents editing, annotations, etc.

While SADL provides a useful way of authoring and testing models, it does not currently provide an easy mechanism for creating visualizations of scenarios and graphical representations of the Subjective Logic values of instances of DPInfo and FigureOfMerit. To do the latter, we implemented an Excel client user-interface with the desired graphical representations. We put together a representative scenario to demonstrate the flexibility and usefulness of our approach. From the scenario and from the user supplied input values, a situation-specific instance data model in the form of an OWL n-triple file is automatically created by the client. This model is passed to a Jena reasoner which reasons over both the logic of the formal model, e.g., transitive closure over class hierarchy, and over the domain rules to create an inferred model. The client then passes a SPARQL query to the reasoner to retrieve the desired information from the inferred model. It uses this information to populate the iconic graphical representations of the DP attributes of simple Messages and the Figure Of Merit rollups of complex Messages.

\section{Analyzing the Records}

We first assigned values to the various cryptographic algorithms. Generally, the stronger a cryptographic function is, the stronger the belief that it provides protection. The weaker a cryptographic function is, the greater the uncertainty whether someone has defeated the algorithm. For instance, any message that is signed with Message Digest 5 (MD5) is given a low belief and high uncertainty because of the recent demonstrations of MD5's defeatability [3]. If we had knowledge that the account was compromised, then we would assign a high value to disbelief of the message's attribute, and the belief and uncertainty values would decrease in response.

Knowledge of the individual information assurance attributes enables better decisions that can adapt to different situations. For instance, if a warfighter was told to investigate evidence that a terrorist was seen entering a building, knowledge of the source of information could affect their reaction to unexpected events. If the source of information was not over a confidential channel, then the warfighter's arrival may be anticipated. If the message has low integrity, then the information could have been distorted and may have to be verified. If the information was not strongly authenticated, then the information may come from an unreliable source. Because of this, we decided to calculate each of IA attributes individually, rather than use a single value to indicate trust. We then created a visual summary of the IA values, to assist in the decision process. 


\subsection{Integrity}

To verify the integrity, first the hash is verified to correspond to the message body. In addition, when the message enters the system, the hash can be compared with the most recent hash. The resulting value is based on the strength of the weakest hash algorithm used.

If the hash values differ when traveling through a person or device, it would indicate a man-in-the-middle attempt. If, for a single hop, the incoming and outgoing hash values differ, then the entity forwarding the message modified it. If the hash differs in a single record, then a substitution occurred during the transmission process.

\subsection{Authenticity}

If the creator of the message signs the initial DP record, authenticity can be based on the algorithmic strength used to sign the record. We should further adjust these values based on the strength of the algorithms used by the certificate authorities. As others have done this [4], we focused on the core attributes, which could be further adjusted based on more subjective opinions (which Subjective Logic can provide).

If the message enters the system unsigned, one of the parties forwarding the message can sign the message. This entity can become a proxy for the sender's ID. Belief of authenticity then becomes subjective based on confidence in the proxy.

\subsection{Confidentiality}

There is no easy way, using just the DP records, to determine if some person ordevice has revealed information using other communication channels. However, we can lower the confidence value every time some entity received a message that they could read. Essentially, the more that know a secret, the less confidence there is in the confidentiality.

We can also base the calculation on the encryption properties of each transmission. If any single transmission is sent unencrypted, the belief in the confidentiality of the message drops to zero, and the uncertainty approaches one.

If the sender and receiver's belief in the confidentiality differ, then this may indicate a system problem. For instance, if a sender believes a message is confidential, but the receiver believes the message has no security, this may indicate an implementation flaw.

\subsection{Non-repudiation}

Non-repudiation is provided for when the sender signs the DP record, which includes the signature of the message author.

\subsection{Availability}

There is no way to calculate the availability of the information, based on just the DP records. This requires knowledge of system characteristics and past history. Given synchronized timestamps, and knowledge of the frequency and expected latency of the messages, is it possible to detect attacks that delay or prevent messages from being received. 


\section{Summarizing Information with an Icon}

When summarizing a complex message with multiple components, there are two different issues to be resolved. The first is the analysis of a simple message from beginning to end. We described how we addressed this in the previous section.

The second issue is summarizing a message containing multiple messages, photos, etc. There are several different consensus functions one can use with Subjective Logic [4]. We considered them, but these make the most sense when dealing with the truthfulness of the content of messages. Since we are only addressing the communication channel, and we ignore the semantic meaning of the messages, we chose a minimum function that summarized a message based the lowest valued attribute of the subcomponents.

For the visualization, we use a simple 3-column icon that shows Confidentiality, Integrity, and Authenticity, with green, red, and gray values representing belief, disbelief, and uncertainty respectively. An example is shown in Figure 3, which uses the colors from top to bottom of green, red, and grey.

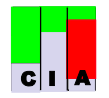

Fig. 2. Icon of Information Assurance Attributes

This example icon shows somewhat high belief in confidentiality, less belief in the integrity, and for illustrative purposes, disagreement on the authenticity of a message as both green and red is shown. Our implementation did not capture any conflict, as it was measuring objective information gathered during the distribution process.

\section{Sample Visualization}

In a sample demonstration, we have 6 messages from five sensors being referenced and included by two analysts, who in turn forwarded their information to a third analyst. The final report consists of 6 images, and the output from the first two analysts. In this workflow, none of the messages are modified. The workflow is shown in Figure 4.

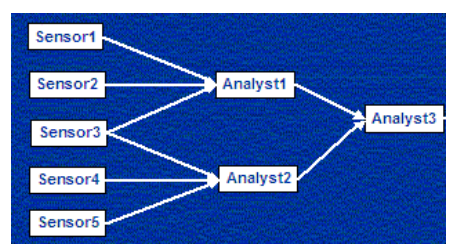

Fig. 3. Sample Workflow 
The GUI is built on top of Microsoft Excel, which also generated the icon. The algorithms used for each of the Information Assurance attributes for each of the sensors, and the reports from the analysts, are selectable. Authenticity is determined by the strength of the algorithm the creator uses to sign the message. The integrity is determined by the strength of the algorithm to sign the Dpinfo record. The confidentiality of the message is determined by the strength of the algorithm used to encrypt the message. In addition, we can introduce security failures in the demonstration, such as invalid signatures, and incorrect hash values. After generating a set of messages corresponding to the conditions of the scenario, the resulting Figure of Merit (FoM) is shown for each of the messages in Figure 5. We assume that each person and device sends information in the most secure way. Because of this, on each outgoing message each of the senders assigned high confidence in their information, and the icons are all green. The receiver may downgrade the trust if stronger encryption algorithms are possible. If any of the cryptographic verifications fail, belief becomes zero, and disbelief is increased, showing red in the FoM icon.

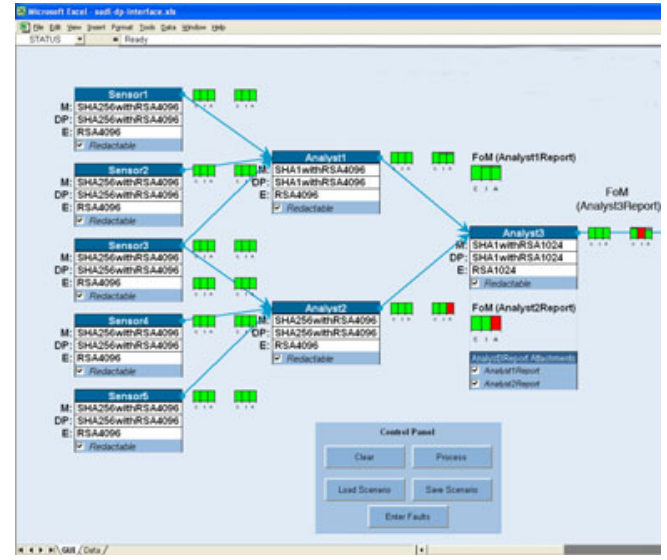

Fig. 4. Sample Results

In Figure 5, we show a sample workflow with 2 failures introduced: Analyst 2's report had an invalid signature (the authentication column is red), and Analyst 3's report indicates a hash mismatch (the integrity column is red), perhaps indicating a man-in-the-middle attack. Note how the icons allowed someone to quickly spot any potential problems. Also note how the authenticity of Analyst 3's report has some uncertainty (some grey on the top of the authentication column), as the signature used the weaker RSA1024 instead of RSA4096.

\section{Conclusion}

We believe that the framework described in this paper is suitable for capturing, summarizing, and analyzing objective evidence about the information assurance attributes of a message at various points in its life cycle, from creation to final destination. We believe 
that measuring the information assurance attributes of the infrastructure is an essential component in measuring the overall trust in a more complex system. We also believe the approach to be suitable for including more subjective information such as opinions and knowledge of outside conditions. Subjective logic provides a well-founded mechanism to resolve conflict and to rollup summary values. The iconic summary is a suitable mechanism to visually display the information assurance attributes of messages and figures of merit, and allows one to quickly identify weaknesses in the communication infrastructure.

Acknowledgements. This paper was prepared by GE Global Research as an account of work sponsored by Lockheed Martin Corporation. Information contained in this paper is the property of Lockheed Martin Corporation. Neither GE nor Lockheed Martin Corporation, nor any person acting on behalf of either; (a). Makes any warranty or representation, expressed or implied, with respect to the use of any information contained in this paper, or that the use of any information, apparatus, method, or process disclosed in this paper may not infringe privately owned rights; or (b). Assume any liabilities with respect to the use of, or for damages resulting from the use of, any information, apparatus, method, or process disclosed in-this paper."

\section{References}

1. Moitra, A., Barnett, B., Crapo, A., Dill, S.: Data Provenance Architecture to Support Information Assurance in a Multi-Level Secure Environment. In: MILCOM 2009, Boston (2009)

2. Jøsang, A.: Artificial Reasoning with Subjective Logic. In: Proceedings of the Second Australian Workshop on Commonsense Reasoning, Perth (1997)

3. Sotirov, A., Stevens, M., Appelbaum, J., Lenstra, A., Molnar, D., Osvik, D.A., de Weger, B. (2008-12-30).: MD5 considered harmful today, http://www.win.tue.nl/hashclash/rogue-ca/ (retrieved December 30, 2008) Announced at the 25th Chaos Communication Congress

4. Jøsang, A.: An Algebra for Assessing Trust in Certification Chains. In: Proceedings of the Network and Distributed Systems Security Symposium, NDSS 1999 (1999)

5. Josang, A.: The Consensus Operator for Combining Beliefs. Artificial Intelligence Journal 141(1-2), 157-170 (2002),

http: //persons.unik.no/josang/papers/Jos2002-AIJ.pdf

6. Crapo, A.: Semantic Application Design Language,

http://sadl.sourceforge.net/ 